\title{
Neonatal Immune Neutropenia due to Isoantibodies against the Granulocyte Receptor Fc $\gamma$ RIIIb
}

\author{
Beatriz Valle Del Barrio $^{a}$ Silvia Maya-Enero ${ }^{a} \quad$ J.J. Rodríguez-Sevilla ${ }^{b}$ \\ Carme Canals Surís $^{c}$ Alba Bosch Llobet ${ }^{d}$ María Ángeles López-Vílchez ${ }^{a}$ \\ ${ }^{a}$ Department of Neonatology, Service of Pediatrics, Hospital del Mar, Universitat Autònoma de Barcelona, \\ Barcelona, Spain; b Service of Hematology, Hospital del Mar, Universitat Autònoma de Barcelona, Barcelona, Spain; \\ ${ }^{\mathrm{C}}$ Immunohematology Laboratory, Blood and Tissue Bank, Barcelona, Spain; ${ }^{\mathrm{d}}$ Transfusion Service-Hospital del Mar \\ and Blood and Tissue Bank, Barcelona, Spain
}

\section{Keywords}

Neonatal alloimmune neutropenia $\cdot$ Neonatal isoimmune neutropenia $\cdot$ Anti-neutrophil antibodies $\cdot$ Fcy receptor IIIb

\begin{abstract}
Introduction: Neonatal neutropenia is often secondary to sepsis, low birth weight, pregnancy-induced maternal hypertension, and other conditions. Case Report: We report a case of asymptomatic isoimmune neutropenia in a pair of preterm twins. Genotyping confirmed that the mother was negative for HNA-1a, 1b, and 1c, consistent with an FcyRIIIb deficiency. The father was $1(a+b+c-)$ and the neonates were $1(a-b+c-)$. A strongly positive result was observed in the granulocyte immunofluorescence test against paternal neutrophils (IgG antibodies). IgG anti-CD16b isoantibodies were detected in the mother's breast milk. Neutropenia resolved after 28 days without requiring any specific treatments. Discussion: Even though neonatal alloimmune neutropenia (NAN) is usually benign and self-limiting, some patients present with delayed separation of the umbilical cord, mild skin infections, omphalitis, or severe infections like pneumonia, sepsis, and meningitis. Thus, it is important to rule out NAN in case of neonatal neutropenia. @ 2021 The Author(s)
\end{abstract}

Published by S. Karger AG, Basel

\section{Introduction}

Neutropenia is defined as an absolute neutrophil count $(\mathrm{ANC})<1.5 \times 10^{9} / \mathrm{L}[1]$, although the reference range varies depending on gestational age, birth weight, gender, and race [2]. It is severe if $\mathrm{ANC}$ is $<0.5 \times 10^{9} / \mathrm{L}$ [3]. Neonatal neutropenia is common, usually transitory, asymptomatic, and secondary to sepsis, prematurity, neonatal alloimmune neutropenia (NAN), low birth weight, pregnancy-induced hypertension, or severe hemolytic disease of the fetus and newborn (HDFN) $[1,4,5]$. Sometimes, it is difficult to distinguish if sepsis preceded neutropenia or vice versa. NAN usually presents with monocytosis compensating the neutropenia, but hemoglobin and platelets remain normal $[4,5]$. Neutropenia frequently exists at birth and may decrease during the first week of life [4]. Clinicians should consider further evaluation of neutropenia if no clear cause is present, or if ANC does not increase within 3-5 days or persists for $>2$ days $[1,2,4]$.

\section{Case Report}

We report 2 cases of NAN due to anti-Fc $\gamma$ RIIIb isoimmunization in a pair of dichorionic female twins born at $35^{4 / 7}$ weeks of gestation from a healthy, nonconsanguineous, 30 -year-old mother with 3 previous healthy children. They weighed 1,980 and 1,748 g (i.e., the 36 and 18 th percentile according to Spanish growth curves for twins). Routine blood tests $12 \mathrm{~h}$ after birth due to a risk of in- 
Fig. 1. GIFT results observed in the crossmatch of the maternal serum against paternal granulocytes, with an FITC-conjugated anti-human IgG anti-globulin. FITC is measured over the population of viable granulocytes, not stained with 7-amino-actinomycin D (Gate P3). MFI, median fluorescence intensity.

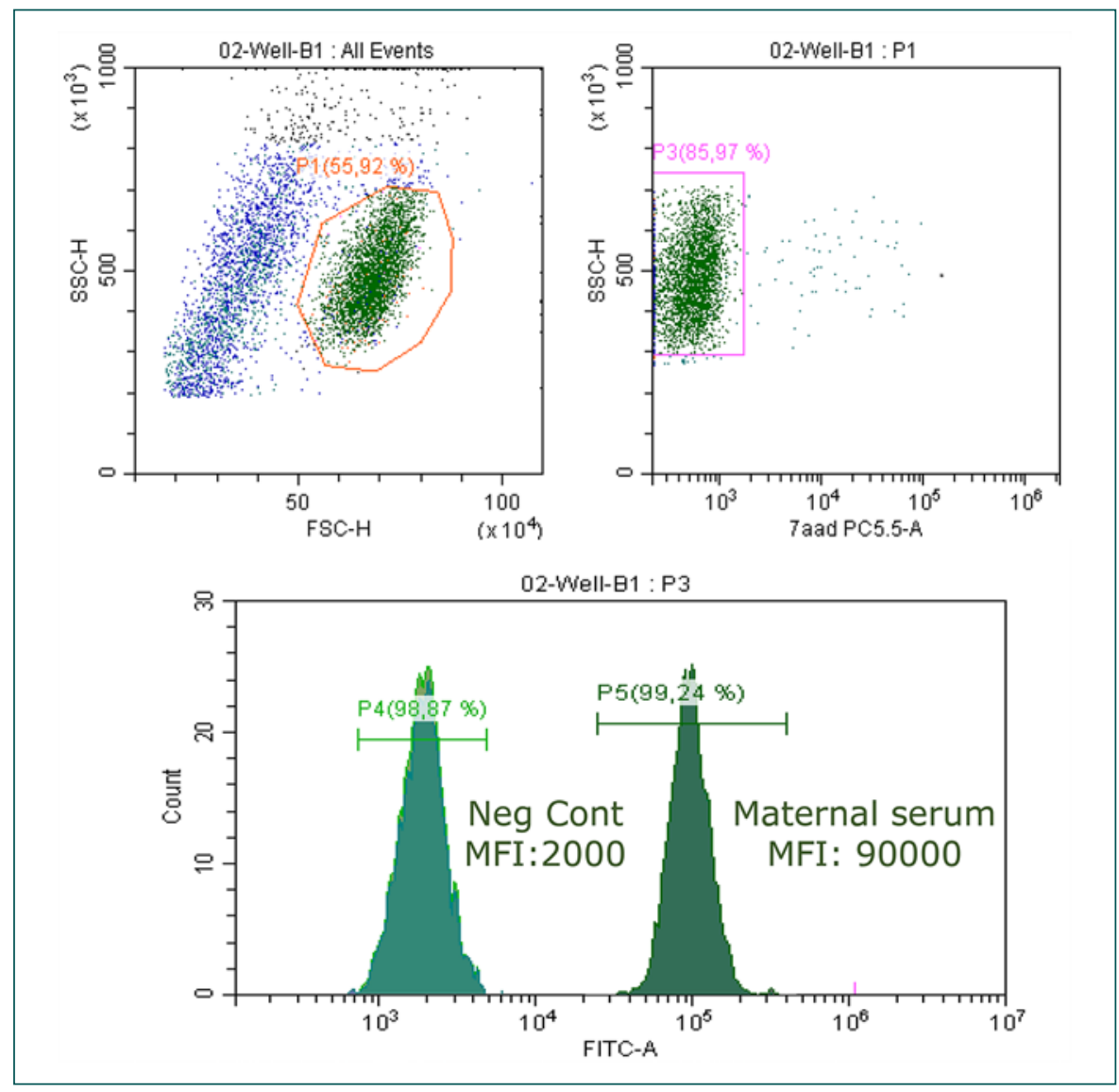

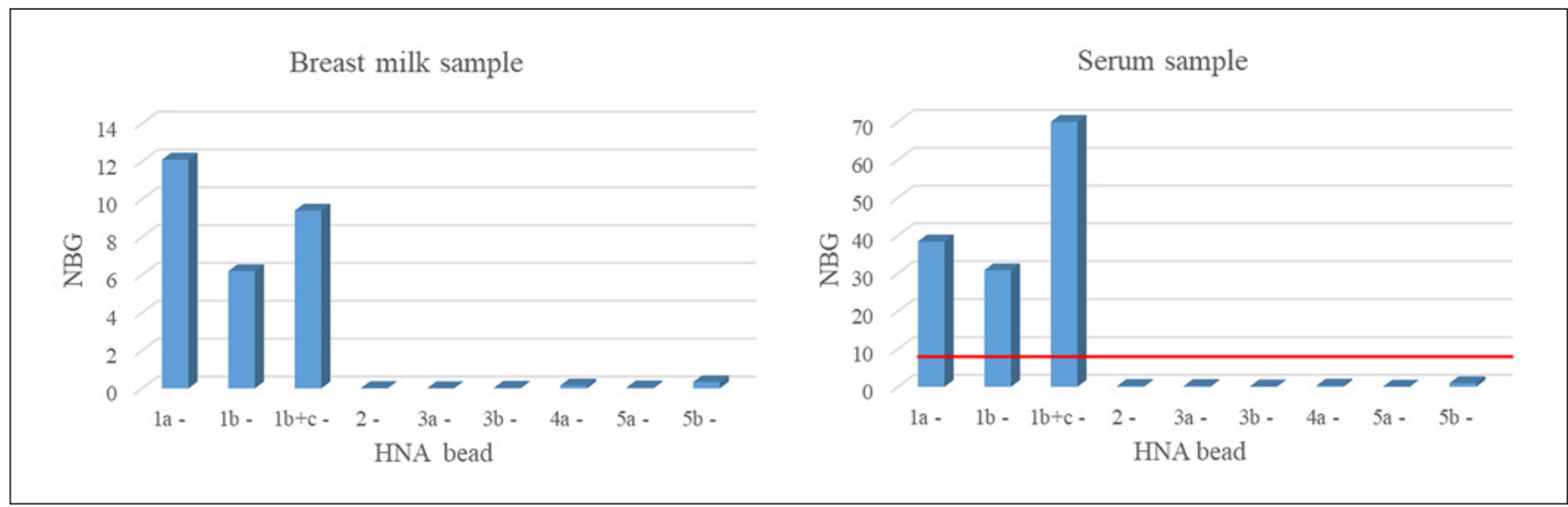

Fig. 2. Results observed in the LABScreen Multi assay for IgG HNA antibody investigation. The red line indicates the standard cut-off value of $>5$ used for serum samples. NBG, normalized background ratio.

fection for group B Streptococcus showed leukopenia and severe neutropenia: a total white blood cell (WBC) count of 6.740 and $4.820 \times 10^{9} / \mathrm{L}$, and ANC 0.067 and $0.048 \times 10^{9} / \mathrm{L}$, respectively. Other hematological and biochemical profiles were normal. Neutropenia was thought to be secondary to early-onset neonatal sepsis. They received antibiotics despite being asymptomatic until blood and CSF cultures were negative. The presence of neutrophil antibodies in the maternal serum was tested with a granulocyte immunofluorescence test (GIFT), granulocyte agglutination test (GAT), and MAIGA (monoclonal antibody-specific immobilization of granulocyte antigen) assay, with fully concordant results. For the
GIFT, GAT, and MAIGA studies, freshly isolated HNA-typed donor cells were used. Cross-matching with the granulocytes of the father could only be performed by the GIFT. For the GIFT assays, an FITC-conjugated $\mathrm{F}\left(\mathrm{ab}^{\prime}\right)_{2}$ fragment goat anti-human IgG antiglobulin (Jackson Immunoresearch, Laboratories Inc.) was used (Fig. 1). The IF results were assessed by flow cytometry (FACSCalibur platform. Becton Dickinson). A strong positive result was observed in the cross-match with the paternal granulocytes, as well as against all the panel cells used, except against granulocytes from a woman with an Fc $\gamma$ RIIIb deficiency. The GAT was performed by standard methods against a panel of granulocytes, and the results 
were negative. The anti-Fc $\gamma$ RIIIb antibody present in the maternal serum did not induce agglutination. Two monoclonal antibodies against the Fc $\gamma$ RIIIb (CD16) were used in the MAIGA: DJ130c (Novus Biologicals) and LNK16 (Invitrogen). The results were in agreement with those observed with GIFT and the Luminex assay LABSCreen Multi. Maternal serum and breast milk were tested with the LABScreen multiassay, including HLA and human neutrophil antigen (HNA) antibody screening by Luminex technology: LABScreen ${ }^{\mathrm{TM}}$ Multi (One Lambda, Inc. CA, USA). Maternal neutrophils were negative for HNA-1a and $1 \mathrm{~b}$, while $50 \%$ expressed HNA-2. Maternal HNA genotyping performed with a multiplex polymerase chain reaction (PCR) for rapid simultaneous detection of all relevant human neutrophil antigens confirmed that the mother was negative for HNA-1a, $1 \mathrm{~b}$, and $1 \mathrm{c}$, consistent with an Fc $\gamma$ RIIIb gene deficiency. The father was $1(\mathrm{a}+\mathrm{b}+\mathrm{c}-)$, and the neonates were $1(\mathrm{a}-\mathrm{b}+\mathrm{c}-)$. HLA antibodies were not detected. Anti-CD16b isoantibodies were detected by GIFT and MAIGA against freshly isolated HNA-typed donor cells and by Luminex. IgG anti-CD16b isoantibodies were detected in the mother's breast milk. As expected, the fluorescence intensity obtained with the breast milk sample was lower than that observed with the serum sample, as the concentration of IgG in human milk is much lower. Nevertheless, as seen in Figure 2, the normalized background ratio (NBG) observed in the milk sample clearly showed a pattern of reaction against the beads carrying the $\mathrm{F} c \gamma \mathrm{RIIIb}$ glycoprotein.

ANC gradually increased without requiring any specific treatments. The neonates were discharged on day 10 with a WBC count 10.25 and $5.04 \times 10^{9} / \mathrm{L}$, and ANC 0.67 and $0.62 \times 10^{9} / \mathrm{L}$, respectively. Neutropenia persisted for 28 days (Table 1). Clinical followup was done until the age of 7 months.

\section{Discussion}

There are 3 types of immune-mediated neutropenia: alloimmune, isoimmune, and autoimmune $[1,4]$. NAN is caused by the maternal sensitization against an antigen of fetal neutrophils inherited from the father $[2,6,7]$. The most frequently involved granulocyte antibodies are those from the HNA-1 family (HNA1a, 1b, 1c, and 1d) that represent polymorphisms of the Fc $\gamma$ RIIIb or CD16b, and HNA2, 3a, 4a, 4b, and 5a [1-4]. NAN can also be caused by antibodies against the Fc $\gamma$ RIIIb in mothers with a Fc $\gamma$ RIIIb deficiency, resulting in the HNA-1 null phenotype, or by HLA class I antibodies against paternal antigens $[2,4,5,8]$. An immunization against an entire glycoprotein that the patient lacks is defined as an isoimmunization (i.e., $\mathrm{RhD}$ isoimmunization in $\mathrm{RhD}$-negative women, who lack all the RhD glycoprotein). An alloimmunization is caused by an antibody against a specific antigen, e.g., an anti-HNA-1a or an HNA-1b. It is often used indiscriminately (commonly for platelet antagonism, not isoimmune neonatal neutropenia). In some cases, NAN is caused by isoantibodies, if a mother lacks a complete HNA system-carrying structure (i.e., antiFc $\gamma$ RIIIb isoantibodies or HNA-2 antibodies). However, there may be some confusion about which name can be best used for this condition [4]. Diagnosis requires identifying the antibody in the maternal serum or in the pa-
Table 1. Trend of peripheral blood leukocytes and neutrophils $\left(\times 10^{9} / \mathrm{L}\right)$

\begin{tabular}{lrrrrr}
\hline \multirow{2}{*}{ Day of life } & \multicolumn{2}{l}{ Leukocytes } & & & \multicolumn{2}{l}{ Neutrophils } \\
\cline { 6 - 6 } & twin 1 & twin 2 & & twin 1 & twin 2 \\
\cline { 1 - 2 } 0 & 6.740 & 4.820 & & 0.067 & 0.048 \\
1 & - & 4.750 & & - & 0.043 \\
2 & 3.410 & 7.820 & & 0.160 & 0.782 \\
4 & 6.110 & - & & 0.305 & - \\
5 & - & 9.680 & & - & 1.064 \\
7 & 10.250 & 3.280 & & 0.820 & 0.426 \\
10 & 10.250 & 5.040 & & 0.670 & 0.620 \\
14 & 10.750 & 9.430 & & 0.752 & 0.471 \\
21 & 8.860 & 8.730 & & 0.088 & 0.349 \\
28 & 10.420 & 9.090 & & 1.810 & 1.640 \\
47 & 7.070 & 10.490 & & 1.050 & 1.580 \\
\hline
\end{tabular}

Normal leukocytes: $9-30\left(\times 10^{9} / \mathrm{L}\right)$. Normal neutrophils: $1.5-$ $5.4\left(\times 10^{9} / \mathrm{L}\right)$.

tient's neutrophils $[6,7]$. The study should be done as soon as possible because antibody titers decrease as time goes by [3]. It is also important to consider that all women with antibodies against HNA antigens may not be used as blood donors due to the potential risk of transfusionrelated acute lung injury (TRALI).

The reported incidence of NAN is 2 per 1,000 live births $[3,5-7,9]$, although it is not exactly known $[2,4]$ because anti-HNA antibody screening and identification assays are laborious [4]. NAN frequently goes undiagnosed, since blood tests are not routinely performed in newborns $[3,5-7,9]$ and it is often detected when WBC counts are performed for nonrelated reasons, as in this case report [4]. Sometimes, the neutropenia is attributed to sepsis, prematurity, intrapartum asphyxia, maternal hypertension or pre-eclampsia $[1,6]$. The clinical course of NAN is usually benign and self-limiting. Symptoms vary from none to a delayed separation of the umbilical cord or mild skin infections, and omphalitis, or severe, even life-threatening infections like pneumonia, sepsis, and meningitis $[1,4]$ mostly caused by $S$. aureus, $S$. epidermidis, and E. coli [8].

The main treatment consists of preventing and treating acute infections $[3,4]$. Prophylactic antibiotics or antifungals such as fluconazole can be considered in severe cases $[3,4]$. The use of intravenous immunoglobulin and recombinant human G-CSF is controversial and may be useful in some cases [1-6]. G-CSF can be considered for severe infections, although it has not increased infectionfree survival $[1,4]$. Intravenous immunoglobulin can be used as a second-line treatment for life-threatening infections or very recurrent infections $[1,3,4]$.

Alloantibodies are cleared spontaneously during the first 6 weeks, but neutropenia can last for up to 6 months 
$[1,2,4,5]$. WBC counts should be performed until neutropenia disappears, which usually happens after a decrease in monocytes [5]. In this study, granulocyte-specific antibodies against HNA were detected in both twins and in the maternal sera and breast milk. IgA, IgG, IgM, $\mathrm{IgD}$, and IgE are present in human breast milk, although at lower levels than in the serum, and the absorption of orally transferred immunoglobulins is inefficient [10]. Transmission of clinically significant amounts of maternal IgG anti-KEL via breast milk in nursing murine pups has been previously reported, although no cases of resulting anemia were observed despite intestinal absorption of IgG being more efficient in mice than in humans [10]. The predominant antibody in colostrum and breast milk is secretory IgA (not tested in our laboratory), although secretory IgM and IgG are present as well. There is a theoretical risk of maintained passage of alloantibodies to infants through breast milk, although, to the best of our knowledge, no cases have been reported to date. It has been described in breastfed infants with HDFN (causing prolonged hemolysis) and in the infants of mothers with immune thrombocytopenia (causing persistent thrombocytopenia) $[9,11,12]$. Discontinuation of breastfeeding is not recommended unless the neutropenia becomes unmanageable. Our patients' mother decided to bottlefeed, and we only obtained breast milk to analyze. Thus, we do not know what effect breastfeeding would have had on the neutropenia. Breast milk contains IgG against Fc $\gamma$ RIIIb. Maternal granulocyte antibodies might be the cause of the prolonged neutropenia observed in some cases of NAN.

It is important to diagnose NAN, to be able to choose the best prophylactic and/or therapeutic options, predict the clinical course, and diagnose NAN in future children. The risk of recurrence is very high, and $\mathrm{WBC}$ and $\mathrm{ANC}$ screening should be performed in future neonates $[2,4$, 5]. Further research is warranted to evaluate if maternal granulocyte antibodies present in breast milk may contribute to a prolonged neutropenia in these patients.

\section{Statement of Ethics}

The patients' parents gave us written permission to publish this case report. The patients are not identifiable through this paper.

\section{Conflict of Interest Statement}

The authors have no conflicts of interest to declare.

\section{Funding Sources}

The authors received no specific funding for this work.

\section{Author Contributions}

B.V.D.B. and S.M.-E. drafted the initial manuscript, reviewed the literature, and reviewed and revised the manuscript. J.J.R.-S. wrote the immunohematologic part, critically reviewed the manuscript for important intellectual content, and reviewed and revised it. C.C.S. helped draft the immunohematologic part, and critically reviewed the manuscript for important intellectual content and revised it. A.B.L. reviewed the literature, critically reviewed the manuscript for important intellectual content, and revised it. M.A.L.-V. helped draft the initial manuscript, critically reviewed it for important intellectual content, and revised it.

\section{References}

1 Dale DC. How I manage children with neutropenia. Br J Haematol. 2017 Aug;178(3): 351-63.

2 van den Tooren-de Groot R, Ottink M, Huiskes E, van Rossum A, van der Voorn B, Slomp J, et al. Management and outcome of 35 cases with foetal/neonatal alloimmune neutropenia. Acta Paediatr. 2014 Nov;103(11):e46774.

3 Riera NE, Kantor GL, Khoury M, Nucci RP, Rapetti MC, Aixala M, et al. Autoimmunealloimmune neonatal neutropenia. Serum reactive IgG and neutrophil-specific phenotype detected by flow cytometry. Medicina (B Aires). 2006;66(5):405-14. Spanish.

4 Porcelijn L, de Haas M. Neonatal Alloimmune Neutropenia. Transfus Med Hemother. 2018 Oct;45(5):311-6.
5 Delacour H, Mornand P, Larréché S, Pilo JE, Mérens A, Imbert P. A neonatal agranulocytosis. Clin Chem. 2014 May;60(5):719-22.

6 Romero P, Brussi M, Garcia Alonso L, Díaz E, Cabanillas L, Pérez E. Alloimmune neonatal neutropenia. Treatment with granulocyte colony- stimulating factor. An Esp Pediatr. 1996; 45:536-8. Spanish.

7 Buznego Sánchez R, Viñuela Roldán JE, Couce Pico ML, Saavedra Pereira MJ, Fernández Lorenzo JR, Fraga Bermúdez JM. Alloimmune neonatal neutropenia due to anti-NA1 antibodies. An Esp Pediatr. 1996 Feb;44(2): 176-8. Spanish.

8 Schibler K. Leukocyte development and disorders during the neonatal period. In: Christensen RD, editor. Hematologic Problems of the Neonate. Philadelphia, PA: WB Saunders Co; 2000. pp. 311-342.

9 Lewin S, Bussel JB. Review of fetal and neonatal immune cytopenias. Clin Adv Hematol Oncol. 2015 Jan;13(1):35-43.
10 Santhanakrishnan M, Tormey CA, Natarajan P, Liu J, Hendrickson JE. Clinically significant anti-KEL RBC alloantibodies are transferred by breast milk in a murine model. Vox Sang. 2016 Jul;111(1):79-87.

11 Hauschner H, Rosenberg N, Seligsohn U, Mendelsohn R, Simmonds A, Shiff Y, et al. Persistent neonatal thrombocytopenia can be caused by IgA antiplatelet antibodies in breast milk of immune thrombocytopenic mothers. Blood. 2015 Jul;126(5):661-4.

12 Leonard A, Hittson Boal L, Pary P, Mo YD, Jacquot C, Luban NL, et al. Identification of red blood cell antibodies in maternal breast milk implicated in prolonged hemolytic disease of the fetus and newborn. Transfusion. 2019 Apr;59(4):1183-9. 